Moritz Spiller*, Nazila Esmaeili, Thomas Sühn, Dr. Axel Boese, PD Dr. med. habil. Salmai Turial, Prof. Michael Friebe, Dr. Alfredo Illanes

\title{
Towards an intraoperative feedback system for laparoscopic access with the Veress needle
}

\author{
A preliminary interface based on Surgical Audio Guidance
}

\begin{abstract}
About $50 \%$ of complications during laparoscopy occur when surgical access is created. The Veress needle and proposed technical alternatives do not provide reliable information to support the surgeons in guiding the needle, or the feedback is not clearly perceivable. Based on acoustic emissions, Surgical Audio Guidance (SURAG) proposes a non-invasive and efficient way to enhance the perception of guidance information through acoustic and visual feedback displayed in real-time. This article demonstrates that the developed feedback matches the information about tissue layer crossings provided by force measurements. This indicates that SURAG can provide an effective means to make laparoscopic access more precise and safe, especially in pediatric surgery, where space for placing the needle is minimal.
\end{abstract}

Keywords: Laparoscopic access, needle guidance, surgical audio guidance, acoustic emissions, intraoperative feedback

https://doi.org/10.1515/cdbme-2021-2008

\section{Introduction}

Throughout the last decade, the number and variety of laparoscopic interventions that are conducted robot-assisted has increased substantially [3] and enabled surgeons to perform laparoscopy with higher precision and accuracy $[2$, 15]. In contrast to that, the first step in laparoscopic surgery,

\footnotetext{
*Corresponding author: Moritz Spiller: SURAG Medical GmbH, Magdeburg, Germany and Medical Faculty, Otto-von-GuerickeUniversity, Leipziger Straße 44, 39120 Magdeburg, moritz.spiller@med.ovgu.de

Nazila Esmaeili, Thomas Sühn, Dr. Axel Boese, Dr. Alfredo Illanes, Prof. Michael Friebe: SURAG Medical GmbH, Magdeburg, Germany and Medical Faculty, Otto-von-GuerickeUniversity, Magdeburg.

PD Dr. med. habil. Salmai Turial: Department of Pediatric Surgery at the University Medical Center Magdeburg, Germany.
}

the creation of laparoscopic access, is still performed manually, and the success of the procedure highly depends on the surgeons' individual experience and skills. Consequently, the procedure is associated with considerable risks, such as injury to blood vessels or intra-abdominal organs such as the intestine.

Laparoscopy is a surgical intervention in the abdomen and pelvis conducted through tiny incisions instead of a complete opening of the abdominal wall, leading to quicker recovery times and less pain for the patient than in traditional open surgery. In a first step, carbon dioxide must be introduced into the abdominal cavity to create the field of vision and work for the laparoscopic procedure (laparoscopic access).

For the creation of laparoscopic access the so-called Veress needle is used in about $50 \%$ of cases across all disciplines [10]. The needle's piercing is always "blind" because there is currently no imaging procedure or other procedure providing navigation information for this surgery step. Instead, the surgeons rely on their sense of touch to guide the Veress needle. A decreasing resistance after passing through a tissue layer (tissue layer crossing) and an additional quiet click caused by the needle's spring mechanism serves as a guide. The surgeon feels and counts the different tissue layers when inserting the needle into the abdominal cavity to orientate himself. Due to this subjective and error-prone technique, it repeatedly happens that the needle is not inserted far enough or, even worse, too far.

While the click and the spring mechanism are intended to act as safety mechanisms, the click is not clearly hearable in noisy operating rooms, and the spring mechanism may also be triggered if no tissue layer has been passed. Moreover, a direct relationship between the surgeons' experience and their perception of the feedback was reported [11].

Several technical solutions have been developed to counter the problem. Veress needles with an integrated LED to indicate tissue layer crossing provides more perceivable feedback but are still based on the error-prone spring mechanism [13, 14]. Additionally, optical solutions with an integrated videoscope $[3,13]$ and needles with pressure or 
impedance sensing at their distal end have been proposed $[8$, $12,17]$. The main disadvantage of the described solutions is the sensor technology that is integrated as a fixed component into the distal end of the needle. Consequently, it is inserted into the patients' bodies which requires a significant re-design of the needle, and the needle must be newly produced and then newly purchased by the clinic. Therefore, it is not possible to use instruments already available in the clinic. Furthermore, these are usually relatively expensive disposable solutions, as sterilization is impossible due to the electrical components.

Surgical Audio Guidance [6, 7] has demonstrated to be able to differentiate between Veress needle events in laparoscopic access [16]. The technique is based on acoustic emissions (AE) concerning the mechanical vibrations generated by the interactions between the needle's tip and tissue. An audio sensor is mounted to the proximal end of the Veress needle to acquire these AE. Thereby, the system can acquire information about tool-tissue interactions noninvasively from outside the body, one of the main advantages of this approach. The current development step is concerned with translating the acquired information into meaningful feedback that enables the surgeons to perform laparoscopic entry more precisely and safely.

In this article, a preliminary feedback interface is presented and evaluated using a database of audio insertion recordings. The interface displays events occurring during the Veress needle insertion, such as the puncture of main layers and reaching the abdominal cavity. The events displayed in the feedback interface are compared with events extracted from synchronously acquired force measurements used as the ground truth of the occurring events. Results show that the generated feedback can provide supplementary sensory information for guidance during laparoscopic access. This demonstrates that SURAG could be an effective means to make laparoscopic access more precise and safe, especially in pediatric surgery, where the space for placing the needle is minimal.

\section{Methods}

\subsection{Problem Statement}

Today, laparoscopic access with the Veress needle is performed using a subjective technique where the surgeons' individual experience and skills are the main factors in avoiding intraoperative complications. Consequently, about $50 \%$ of all complications during laparoscopic procedures are caused when surgical access is created $[1,4]$. In some cases, the conversion to open surgery may be necessary to treat injuries that have occurred. Rectification of such injuries can last up to $12 \%$ of the total operating time [9]. Therefore, in addition to the patient's risks and an extended recovery time, Veress needle injuries lead to considerable additional time expenditure and hospital costs, highlighting the need for a guidance technology that enables surgeons to create laparoscopic access more precisely and safely.

The major problem of the Veress needle's safety mechanism is not always perceivable by the surgeons. The click triggered by the Veress needle's spring load is not always hearable and might occur even if no tissue layer is crossed.

Due to that, we hypothesize that surgeons would benefit from an enhanced perception of tissue-layer crossings during laparoscopic access. Our preliminary interface aims to provide that by visual and acoustic feedback.

\subsection{Data Acquisition}

The interface presented in this article was developed based on a dataset acquired during the automated insertion of the Veress needle into a gelatin block filled with ex-vivo porcine fat tissue. This phantom simulates the tissue layers (fascia, fat, muscle) that the Veress needle needs to pass during real-world insertions. A material testing was used to repeatedly insert a $120 \mathrm{~mm}$ long, metallic Veress into the gelatin phantom. The insertion was conducted at a constant speed of $5 \mathrm{~mm} / \mathrm{s}$ in a vertical downward movement while the occurring counteraction forces were measured. A MEMS microphone was mounted via a $3 \mathrm{D}$ printed adapter to the proximal end of the Veress needle to acquire the acoustic emissions that occur during insertion. For more details, please refer to [16].

\subsection{Signal Processing}

The diagram of Figure 1 displays the main steps for the signal processing strategy for providing acoustic and visual feedback. First, the signal is bandpass filtered in order to reduce very low-frequency trends and high-frequency noise. In order to provide the audio feedback, this filtered signal is amplified, and real-time played back through speakers. Finally, an envelope indicator is computed from the filtered signal to enhance the puncture events for visual feedback. This indicator is then translated into an image through a simple color map plot that is finally real-time displayed on an external screen. 
The force signal is processed using the approach presented in [5] to enhance the puncture events used as ground truth events. A puncture appears in the force as a peak in the signal followed by a significant drop-out. The processing of the force intends to enhance these dynamics. For that, a moving average filter followed by a derivative filter is applied to the force signal.

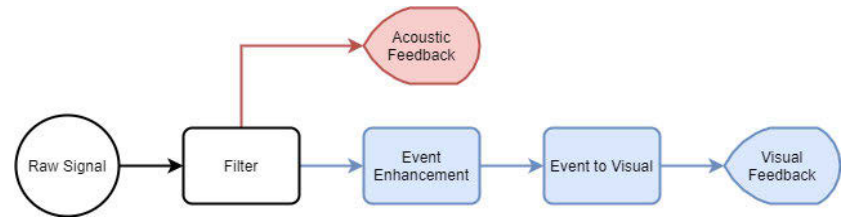

Figure 1: Block diagram of the signal processing strategy.

\section{Results \& Discussion}

Our developed feedback interface is shown in Figure 2. It includes A) the raw audio signal, B) the processed audio signal after event enhancement (see also Figure 1), and C) the visual feedback.

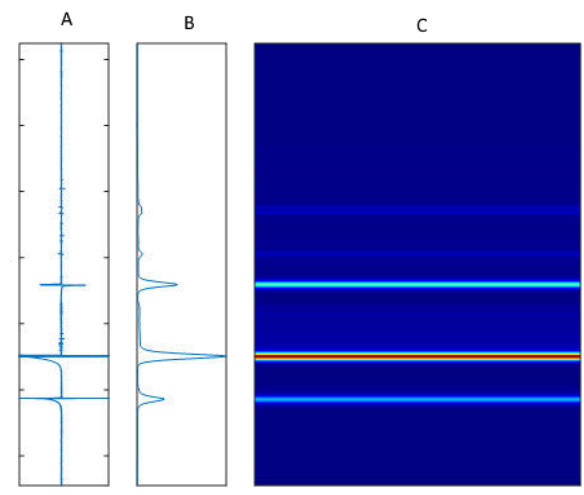

Figure 2: The visual feedback for Veress needle guidance. The insertion displayed here corresponds to the audio and force signal presented on the left column of Figure 3.

The visual feedback consists of a color map plot (see also Section 2.3) where dark blue areas represent the passing of the needle through homogenous tissue like fat or muscle. A tissue layer crossing is represented by the horizontal lines in the graph, where each major line indicates the crossing of a tissue layer. Using this straightforward methodology, the surgeons can observe in real-time when the Veress needle passes from one tissue to another. Integrating that information with their anatomical knowledge, surgeons can determine the tissue layer the needle's tip currently is in and if the abdominal cavity has already been reached. This feedback variant also provides history about how many layers have been crossed so far. This may free up the surgeons' mental working memory from counting the resistances that they have perceived and enables them to focus on their actual task. In parallel to the visual feedback, the acoustic feedback can be played via loudspeakers. The acoustic feedback allows the surgeons to focus on the operating area instead of the external screen displaying the visual feedback while still receiving valuable feedback for guiding the needle.

In order to prove that the visual feedback generated on the acquired audio signal displays the actual tissue layer crossings, force measurements were acquired during the experiment (see also Section 2.2). Two of the acquired force signals (column $\mathrm{A}$ and $\mathrm{B}$ ) are displayed in Figure 3, where the raw force signal (line 3 ), the force derivative (line 2) and a combination of the raw audio signal with the audio signal after event enhancement (line 1) are compared to each other. Each significant drop in the force signal (line 3 ) corresponds to a reduction of the necessary force to insert the needle further, therefore the breakage of tissue and a tissue layer crossing. The force derivative (line 2) highlights those force reductions, and it can be observed that the events in line 1 match the peaks in the force derivative and therefore indicate an actual tissue layer crossing.
A

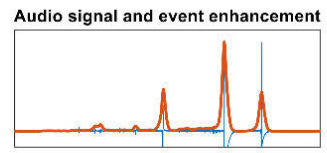

1
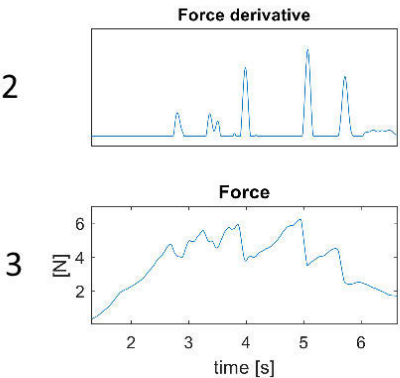

B
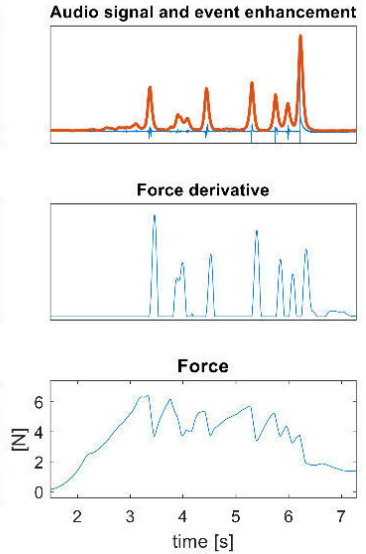

Figure 3: Two audio signals acquired during Veress needle insertion into two different tissue phantoms compared to the respective force measurements.

While only the visual feedback is relevant to the surgeons during Veress needle insertion, the raw audio signal and the audio signal after event enhancement were added to the interface for testing purposes and demonstrate that the visual feedback matches the acquired audio signal. Since this might impair usability, the unnecessary components will be removed in the further development process. Furthermore, the feedback should be displayed in the surgeons' field of view during the procedure instead of a separate screen to enable them to focus on the operating area during insertion.

While the force measurements provide similar information as the audio signal, our audio-based approach is 
more cost-efficient and easier to use since it requires only a low-cost microphone instead of an expensive force sensor. Therefore, we use the force signal only as a reference to show that the audio signal contains valuable information about the process of inserting the Veress needle.

\section{Conclusion}

This article presents the preliminary version of an intraoperative feedback system that enhances the surgeons' perception of tissue layer crossings during laparoscopic access with the Veress needle. It was shown that the developed visual feedback could reliably display the information contained in the acquired audio signal, which in turn contains the identical information as the force signal about tissue-layer crossings.

This indicates that an intraoperative feedback system based on Surgical Audio Guidance can effectively enhance the surgeons' perception of tissue layer crossing during Veress needle insertion.

\section{Author Statement}

Research funding: This research was partly funded by the German Federal Ministry for Economic Affairs and Energy, EXIST Transfer of Research 03EFOST032, and the European Union, European Social Fund for Germany. Conflict of interest: Authors state no conflict of interest.

\section{References}

Alkatout, I. 2017. Complications of Laparoscopy in Connection with Entry Techniques. Journal of gynecologic surgery 33, 3, 81-91.

Badani, K. K., Bhandari, A., Tewari, A., and Menon, M. 2005.

Comparison of two-dimensional and three-dimensional suturing: is there a difference in a robotic surgery setting? Journal of endourology 19, 10, 1212-1215. Heemskerk, J., Bouvy, N. D., and Baeten, C. G. M. I. 2014. The end of robot-assisted laparoscopy? A critical appraisal of scientific evidence on the use of robot-assisted laparoscopic surgery. Surgical endoscopy 28, 4, 1388-1398. Huang, H.-Y., Yen, C.-F., and Wu, M.-P. 2014. Complications of electrosurgery in laparoscopy. Gynecology and Minimally Invasive Therapy 3, 2, 39-42.

[5] Illanes, A., Boese, A., Friebe, M., and Hansen, C. 2020. Feasibility Check: Can Audio Be a Simple Alternative to ForceBased Feedback for Needle Guidance? In Medical Image Computing and Computer Assisted Intervention - MICCAI
2020, A. L. Martel, P. Abolmaesumi, D. Stoyanov, D. Mateus, M. A. Zuluaga, S. K. Zhou, D. Racoceanu and L. Joskowicz, Eds. Lecture Notes in Computer Science. Springer International Publishing, Cham, 24-33. DOI=10.1007/978-3-030-59716-0_3.

Illanes, A., Boese, A., Maldonado, I., Pashazadeh, A., Schaufler, A., Navab, N., and Friebe, M. 2018. Novel clinical device tracking and tissue event characterization using proximally placed audio signal acquisition and processing. Scientific reports 8, 1, 12070.

[7] Illanes, A., Suhn, T., Esmaeili, N., Maldonado, I., Schaufler, A., Chen, C.-H., Boese, A., and Friebe, M. 2019 - 2019. Surgical

Audio Guidance SurAG: Extracting Non-Invasively Meaningful Guidance Information During Minimally Invasive Procedures. In 2019 IEEE 19th International Conference on Bioinformatics and Bioengineering (BIBE). IEEE, 567-570. DOI=10.1109/BIBE.2019.00108.

[8] Janicki, T. I. 1994. The new sensor-equipped veress needle. The Journal of the American Association of Gynecologic Laparoscopists 1, 2, 154-156.

[9] Jung, J. J., Adams-McGavin, R. C., and Grantcharov, T. P. 2019. Underreporting of Veress Needle Injuries: Comparing Direct Observation and Chart Review Methods. The Journal of surgical research 236, 266-270.

[10] Krishnakumar S, T. P. 2009. Entry Complications in Laparoscopic Surgery. Journal of Gynecological Endoscopy and Surgery, 1(1), 4-11.

[11] Li, X.-L., Du, D.-F., and Jiang, H. 2016. The learning curves of robotic and three-dimensional laparoscopic surgery in cervical cancer. Journal of Cancer 7, 15, 2304-2308.

[12] Ott, J., Jaeger-Lansky, A., Poschalko, G., Promberger, R., Rothschedl, E., and Wenzl, R. 2012. Entry techniques in gynecologic laparoscopy—a review. Gynecological Surgery 9 , 2, 139-146.

[13] Reza S. Mohajer. Veress Needle with illuminated Guidance and Suturing Capability, US 8523817 B2.

[14] Reza S. Mohajer. Veress Needle with illuminated tip and cavity penetration indicator, US $8838206 \mathrm{~B} 2$.

[15] Santiago Horgan and Daniel Vanuno. Robots in Laparoscopic Surgery.

[16] Schaufler, A., Sühn, T., Esmaeili, N., Boese, A., Wex, C., Croner, R., Friebe, M., and Illanes, A. 2019. Automatic differentiation between Veress needle events in laparoscopic access using proximally attached audio signal characterization. Current Directions in Biomedical Engineering 5, 1, 369-371. Yun, J., Kim, H. W., Kim, H.-I., and Lee, J.-H. 2017. Electrical impedance spectroscopy on a needle for safer Veress needle insertion during laparoscopic surgery. Sensors and Actuators B: Chemical 250, 453-460. 